Article

\title{
A Reconfigurable Gripper for Dexterous Manipulation in Flexible Assembly
}

\author{
Jason Spiliotopoulos, George Michalos (1) and Sotiris Makris * \\ Laboratory for Manufacturing Systems and Automation, Department of Mechanical Engineering and \\ Aeronautics, University of Patras, 26504 Patras, Greece; spiliot@lms.mech.upatras.gr (J.S.); \\ michalos@lms.mech.upatras.gr (G.M.) \\ * Correspondence: makris@lms.mech.upatras.gr; Tel.: +30-261-091-0160
}

Received: 9 November 2017; Accepted: 5 January 2018; Published: 10 January 2018

\begin{abstract}
In this paper, a high-speed multi-fingered reconfigurable gripper is presented. The aim is to create a robotic end effector that is capable of handling parts of different geometries and weight. It consists of three fingers, accounting for a total of eight Degrees of Freedom (DoFs). The paper discusses the design, control, and reconfiguration aspects of the gripper, and demonstrates its applicability to different manipulation tasks. The gripper has the ability of high-force movement and its high number of DoFs enables it to grasp a large variety of geometries, while retaining the design simplicity. The preliminary grasping experiments highlight its potential in robotic handling applications in both research and production environments.
\end{abstract}

Keywords: gripper; reconfigurable end effector; robotic manipulation; industrial robot; assembly

\section{Introduction}

In the past years, there has been a vast activity towards the development of reconfigurable resources to increase the flexibility of industrial assembly operations [1]. At the resource level, this flexibility has been sought in multiple forms and levels: control strategies for flexible lines [2], reconfigurable robotic systems $[3,4]$, autonomous mobile manipulators $[5,6]$, cooperating robots $[7,8]$, and advanced perception/sensing technologies [9]. Nevertheless, the ability of the end effectors and tooling to carry out the task has been the main limitation for actually carrying out the manipulation tasks [10]. The vision has been the development of low-cost grippers that present dexterity combined with high speeds and grip forces, yet robust and simple both in its programming and development. To better satisfy some of these requirements, the design of robotic hands has followed two distinct paths. The first one was more industrially oriented, focusing on more robust, simple, and low-cost solutions, while sacrificing dexterity. The second approach has been aiming at achieved higher speed, dexterous grippers at the expense of high complexity and costs.

The first approach has been pursued by most industrial gripper suppliers. Schunk, being one of the leading companies in industrial manipulation, has developed a variety of two finger [11] as well as three finger grippers [12], which have become widely adopted, due to their simplicity in design and control. On a similar concept, while aiming for greater adaptability, RobotIQ has developed two two-finger grippers, with one controlled Degree of Freedom (DOF) and a three finger one with four actuators [13]. The Barrett hand, one of the most commercially successful grippers, can also be placed in this category [14]. It consists of eight joints, which are controlled by four servomotors, is lightweight and yet powerful and dexterous in precise motions. On the other hand, it is limited by its relatively low number of independent DoFs. In a similar direction, several grippers have been presented by the academic community based on the principle of under actuation. The LARM Hand IV [15] is a three finger gripper, actuated by four motors that are embedded in the palm of the hand. Furthermore, 
the Tuat/Karlsruhe Hand [16] is a five-finger hand that consists of only one active DoF. The University of Nazarbayev also presented in 2013 a five-finger gripper whose controlled DoFs had been kept to the low number of four by creating a dependency in the movement of its last three fingers [17]. On a similar concept, Prensilia Srl. developed a five-finger hand, called Azzurra IH2, with five active DOFs, while its two last fingers were forced to open and close at the same time [18].

Highly dexterous grippers consist of a high number of DoFs and mostly use the multigrasp hand paradigm, while being relatively complex and expensive. The Stanford Hand/JPL [19], Utah/MIT dexterous hand [20], and the fourteen degrees-of-freedom Robonaut hand for space operations [21] were among the first high-DoF hands to be developed. The BUAA/Beijing University [22] consists of four fingers with a total of $16 \mathrm{DoFs}$, and the NTU hand [23] consists of a five-finger design, which accounts for an overall of seventeen DoFs. In the Gifu Hand II [24], and III [25] the sixteen actuators required for the movement of its 20 joints have been incorporated inside the fingers as opposed to many of the other grippers. The same principle was also applied to the DLR Hand II [26] consisting of 13 controlled DOFs. The UB Hand IV presented in 2013 [27] has an overall of 20 DOFs, enabling the movement of its five independent fingers. Another one of the most widely known hands for its dexterity, is the Shadow C5 Dexterous Hand [28], developed by the Shadow Robot Company that can reproduce the 26 degrees-of-freedom of the human hand by using 23 DoFs, controlled by remotely placed motors or pneumatic tendons.

Although some grippers aim at satisfying both high reconfigurability and simplicity, most of them are characterized by low gripping forces. SCHUNK presented a gripper called the SDH Hand [29], which consists of three fingers and has seven active DOFs, while the actuators are placed inside the fingers, having a maximum gripping torque of $1.4 \mathrm{Nm}$. Elumotion Ltd. (Bath, UK) has also developed the ELU-2 Hand [30] that has five fingers and an overall of nine DOFs, while being self-contained. However, its fingertip forces are limited to $4.7 \mathrm{~N}$. Manipulation speed has also been a great issue as very limited approaches have managed to combine a large number of DoFs with successful and repeatable high-speed manipulation [31].

The main contribution of this research can be summarized in the following:

- $\quad$ Development of a multi-fingered high-DoF gripper, with anthropomorphic features.

- Simple design and yet capable of grasping parts with varying geometrical and physical properties.

- $\quad$ Providing a good tradeoff between (a) the dimensions and shape of the parts that it can grasp, including highly asymmetric objects, (b) the achieved gripping forces, and (c) the dynamic behavior of the fingers that can move at high speed and accelerations.

This paper is organized as follows. In Section 2, the design of the gripper, including the design principle, the mechanical, and kinematic design, is discussed. In Section 3, the implementation of the previously described design is documented, along with the actuation and control that were integrated. Section 4 is dedicated to discussion and future work.

\section{Materials and Methods}

The design of the discussed gripper has been aimed at satisfying the need for a dynamic, high-speed, reconfigurable gripper that still retains the design simplicity, which is required for robust operation and easy maintenance. Such requirements arise from the manufacturing sector such as the automotive body in white and final assembly stages, where high variation in the parts geometry and weight are observed. Section 3 provides several examples of such cases that have been used as the starting point for the design of this gripper.

Starting with the need for high re-configurability and the ability to accommodate a variety of grasping positions/geometries, a three-finger arrangement with eight DoFs, has been selected. This design uses the ability of the two fingers to enlarge the grippers' grasp capabilities, as discussed in [32]. Generally, for a hand to be able to grasp an object at a random position and orientation, it would require at least nine DoFs, the gripper in this work has been limited to eight DoFs by reducing the 
middle finger's ability to rotate around the base of the hand. This DoF greatly simplifies the design and can be easily compensated through the robotic manipulator where the gripper is attached.

The selection of having eight independent DoFs may seem to contradict the requirement for weight, space, and complexity minimization, however this is partially addressed by the use of very efficient (size to performance ration) of the utilized motors that can be connected and controlled under a single network. The gripper has indeed larger weight and dimensions when compared to existing ones (see also Section 3.1), but the individual actuation of all links allows for grasping highly non-symmetric objects by achieving asymmetric configurations for both the adduction and abduction motions. This benefit, when combined with the ability to handle heavier parts is of vast importance for the case of industrial assembly where such components are quite common (e.g., in automotive or white goods assembly sector). A possible coupling between some of the DoFs could simplify the control, but at the expense of attainable gripper configurations.

When considering the dynamics of the reconfiguration movement, all of the gripper joints have been designed to be able to execute their full movement in $100 \mathrm{~ms}$. This means that any gripper joint can execute 180-degree movement in $0.1 \mathrm{~s}$. The weight of the mechanism is a factor of utmost importance in high-speed applications. For this reason, a limit of $5 \mathrm{~kg}$ has been considered, while still being able to grasp objects weighing a few kilograms.

Finally concluding the requirements for such a gripping system, the control aspects also need to be considered. Thus, a centralized master node to monitor and control all actuators simultaneously has been considered. In this case, the exchange of all the messages to/from the controller should be carried out in real time to enable the required monitoring of the joint movement. Table 1 summarizes the design requirements as discussed above.

Table 1. Gripper design requirements.

\begin{tabular}{|c|c|}
\hline Requirement Type & Description \\
\hline Mechanical & $\begin{array}{ll}\text { - } & \text { Number of fingers: } 3 \\
\text { - } & \text { Degrees of Freedom: } 8 \\
\text { - } & \text { Gripper weight: less than } 5 \mathrm{~kg} \\
\text { - } & \text { DoF speed: } 180 \mathrm{deg} / 0.1 \mathrm{~s}\end{array}$ \\
\hline Sensing & $\begin{array}{ll}\text { - } & \text { Position } \\
\text { - } & \text { Velocity } \\
\text { - } & \text { Force/torque }\end{array}$ \\
\hline Control & $\begin{array}{l}\text { - } \quad \text { Centralized control for all nodes } \\
\text { - } \quad \text { Real time monitoring/control of } \\
\text { all actuators }\end{array}$ \\
\hline Product & $\begin{array}{l}\text { - } \quad \text { Varying geometries } \\
\text { - } \quad \text { Product weight: up to a few kilograms }\end{array}$ \\
\hline
\end{tabular}

\subsection{Design Principle}

The developed gripper is aimed at being reconfigurable for achieving a wide range of grasping modes. A grasp can be mainly categorized either by the position of the fingers (centripetal, parallel), or by the way that the final grasp of the object is achieved (encompassing, pinching). As far as the first category is concerned, Figure 1 depicts how the different grasp modes are achieved by the gripper.

The actual developed gripper is shown in Figure 2. In the centripetal grasp, all of the fingers are placed in a position surrounding a central point in space, while in the case of parallel grasps, a number of fingers are set to a parallel position relative to each other. The encompassing and pinching grasping modes, on the other hand, comprise a classification that mainly lies in the precision of the task and the geometrical characteristics of the object [33]. 
Enveloping grasps are characterized by a multitude of contact points between the object and the gripper's fingers. The object is surrounded by the gripping parts of the hand. This grip is the most powerful and can passively resist arbitrary external forces exerted on the object. Although this grasp is the most stable, it can only be used on objects requiring very low dexterity.

The Pinching grasp, on the other hand, consists of relatively few contact points that are mostly located on the fingertips. On the human hand, in this gripping type, the thumb is placed opposite to the other fingers to provide the required stability. This grip's stability may be easily disturbed and it is subject to the position of the contact points, since their number is very limited in comparison to the encompassing grip.

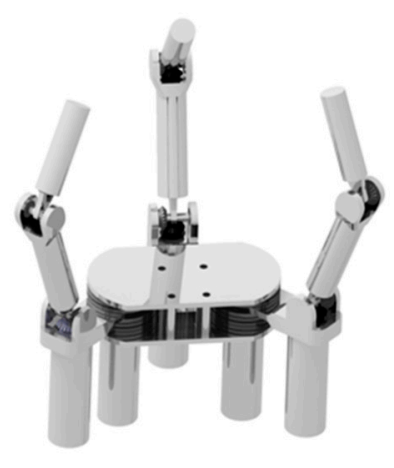

(a)

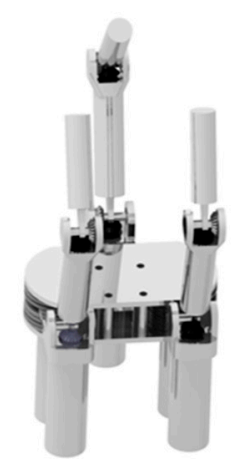

(b)

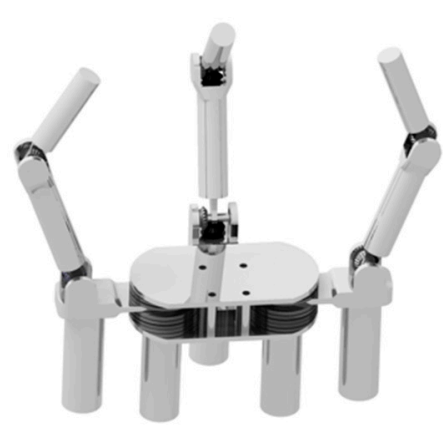

(c)

Figure 1. (a) Three-finger centripetal; (b) 3-finger parallel and (c) two-finger parallel grasping poses.

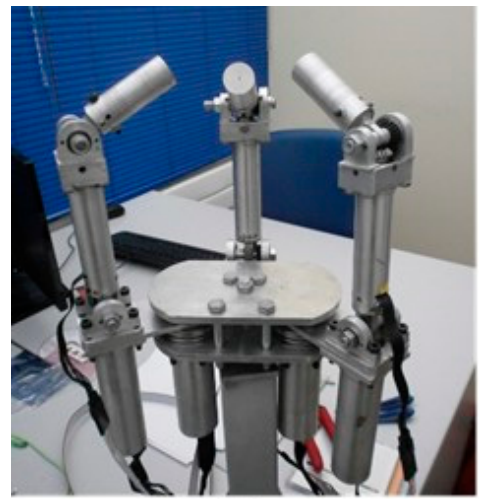

(a)

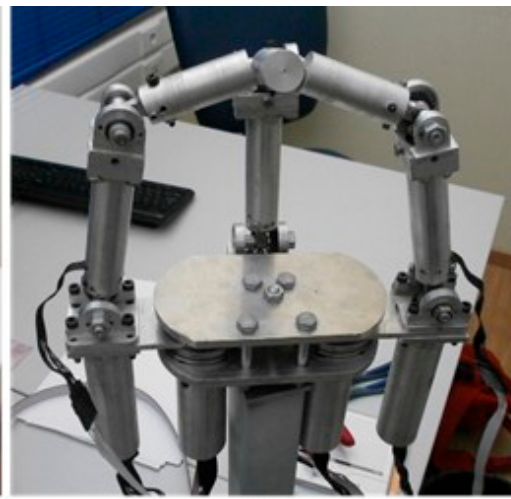

(b)

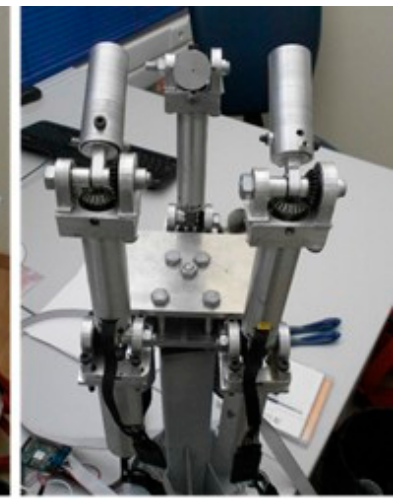

(c)

Figure 2. (a) Three-finger centripetal; (b) two-finger parallel, and (c) three-finger parallel grasping poses achieved by the developed gripper.

Figure 3 shows one of the possible ways that an encompassing grasp can be accomplished by the gripper, as well as a feasible pinching grip. The type of grip, and therefore, the required configuration of the fingers are dependent on the geometry of the object to be grasped. 


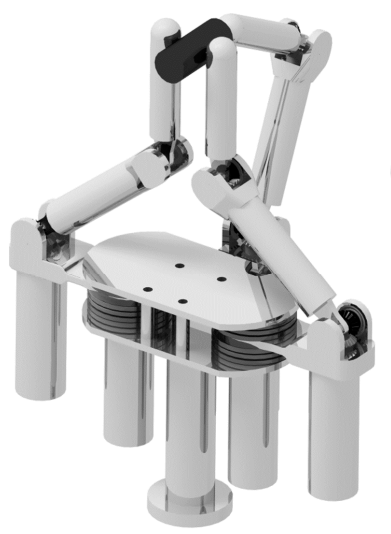

(a)

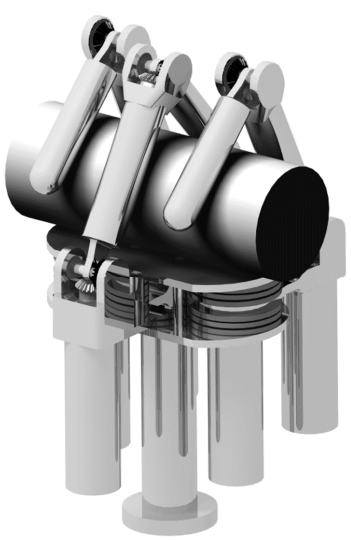

(b)

Figure 3. Simulation of the (a) pinching and (b) encompassing grasp achieved by the designed gripper.

\subsection{Mechanical Design}

When considering the requirements for design simplicity and high-speed movement, simple articulation and transmission mechanisms have been selected. The rotation of the two fingers around the gripper base is achieved by fixing the component that supports the fingers on the gripper base directly on the motor output shaft. In this way, the backlash is drastically reduced, as shown in Figure 4a. In order for this rotation to be as frictionless as possible, the finger assembly interface is supported by two single-direction thrust bearings, placed on the top and bottom of it, as illustrated in Figure $4 \mathrm{~b}$. The actual implemented configuration is demonstrated in Figure $4 \mathrm{c}$ where the thruster bearings below and on top of the connecting element (shown in Figure 4a) are clearly shown.

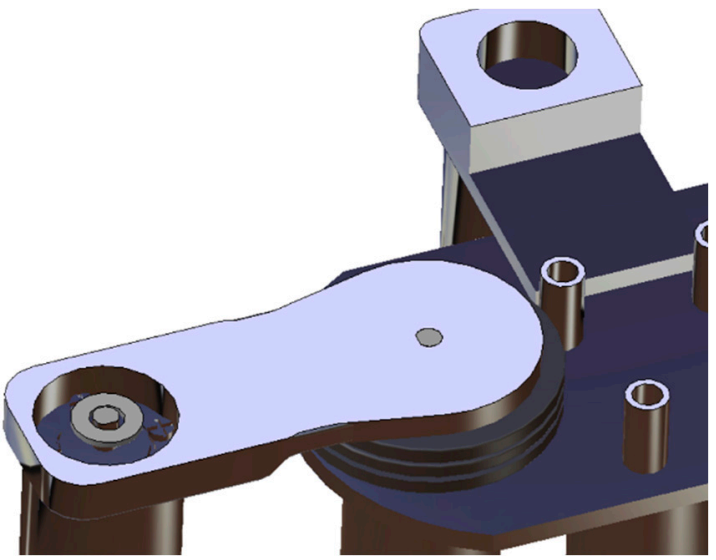

(a)

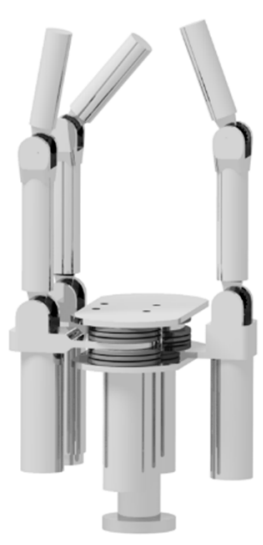

(b)

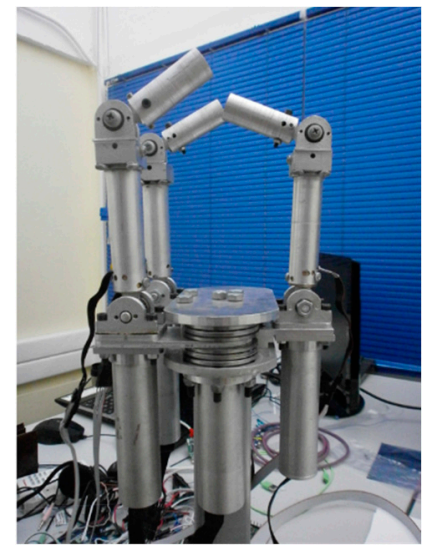

(c)

Figure 4. (a) Gripper design cross section on the finger assembly interface; (b) gripper design and (c) developed gripper depicting the thrust bearings.

For the rest of the gripper joints the motors have been placed inside the body of the fingers and thus the axis of rotation has to be converted from vertical to horizontal (relative to the motor's output shaft). For this reason, a pair of straight bevel gears is chosen, as demonstrated in Figure 5 . One of these is fixed on the motor output shaft and the other one is at a relative 90 degrees position, fitted inside a ball bearing. In the center of this bevel gear, the joint axle whose motion is supported is fixed supported by another ball bearing in the other end. 


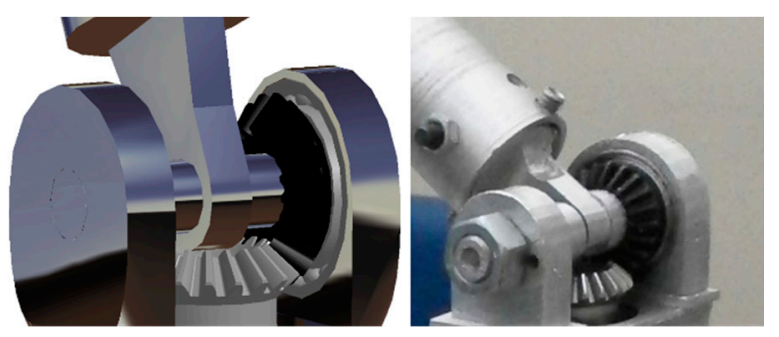

(a)

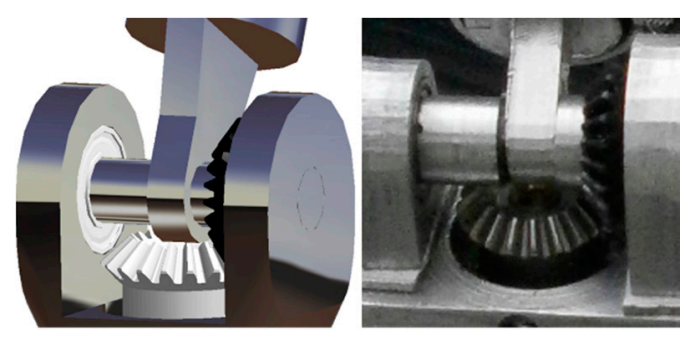

(b)

Figure 5. Design and actual joint views: (a) axle to bevel assembly and (b) axle to bearing.

The direct connection of the motor shaft with the phalanges, although providing an immediate response, may be considered as a weak point as it can lead to motor damage in the case of impact loads on the fingers. As the finalization of the gripper end tips is ongoing and field tests will be carried out to assess the operating environment of the gripper (forces, loads, acceleration etc.), the development of compliant motor control schemes and the integration of elements to absorb the extra forces without compromising positioning accuracy is proposed as future work. Under the same scope, dedicated experiments will be required to prove the ability of the individual elements to absorb collisions.

\subsection{Kinematics}

The kinematic problem of a mechanism can be approached either with the use of forward or inverse kinematics. Forward kinematics is a set of equations that can define the frame of the end-effector, as a function of the joint angles. Inverse kinematics, on the other hand, define the joint parameters of a desired end-effector frame position. In the latter case, one can get a possible number of joint angles for a specific coordinate frame. Although some of the solutions may be mathematically correct, it is not guaranteed that they correspond to attainable physical solutions. Consequently, each set of solutions should be first checked to determine whether it is in agreement with the physical link limits.

In this case, to also obtain the inverse kinematics of the gripper, each of the fingers has been viewed as a serial manipulator with its base at the first joint axis. After the forward kinematics of the serial manipulator has been calculated, a transformation is calculated to consider the difference between the base of each finger and the user's frame of reference (the frame on the center of the base of the gripper). The main idea of this approach is similar to a modular radially symmetric six-legged robot presented in [34]. The forward kinematics analysis has been carried out using the Denavit-Hartenberg (DH) parameters [35], as presented in Table 2 for one of the three-DoF fingers.

Table 2. Denavit-Hartenberg (DH) parameters for one of the three Degrees of Freedom (DoF) fingers.

\begin{tabular}{ccccc}
\hline Link & $\boldsymbol{\theta}_{\mathbf{i}}$ & $\boldsymbol{\alpha}_{\mathbf{i}-\mathbf{1}}$ & $\mathbf{a}_{\mathbf{i}-\mathbf{1}}$ & $\mathbf{d}_{\mathbf{i}}$ \\
\hline 1 & $\theta_{1}$ & 0 & 0 & 0 \\
2 & $\theta_{2}$ & $-\pi / 2$ & $\alpha_{2}$ & $\mathrm{~d}_{2}$ \\
3 & $\theta_{3}$ & 0 & $\alpha_{3}$ & 0 \\
4 & $\theta_{4}$ & 0 & $\alpha_{4}$ & 0 \\
\hline
\end{tabular}

A generic transformation matrix has the following form [36].

$$
{ }_{i}^{i-1} T=\left[\begin{array}{cccc}
c \theta_{i} & -s \theta_{i} & 0 & a_{i-1} \\
s \theta_{i} c \alpha_{i-1} & c \theta_{i} c \alpha_{i-1} & -s \alpha_{i-1} & -s a_{i-1} d_{i} \\
s \theta_{i} s \alpha_{i-1} & c \theta_{i} s \alpha_{i-1} & c \alpha_{i-1} & c a_{i-1} d_{i} \\
0 & 0 & 0 & 1
\end{array}\right]
$$


where, $c \theta_{i}, s \theta_{i}$, stand for $\cos \theta_{i}$ and $\sin \theta_{i}$ respectively represent the transformation matrices from $i-1$ to the $i \mathrm{DoF}$ and can be calculated as shown below.

$$
\begin{gathered}
{ }_{1}^{0} T=\left[\begin{array}{cccc}
c \theta_{1} & -s \theta_{1} & 0 & 0 \\
s \theta_{1} & c \theta_{1} & 0 & 0 \\
0 & 0 & 1 & 0 \\
0 & 0 & 0 & 1
\end{array}\right], \\
{ }_{2}^{1} T=\left[\begin{array}{cccc}
c \theta_{2} & -s \theta_{2} & 0 & \alpha_{2} \\
0 & 0 & 1 & d_{2} \\
-s \theta_{2} & -c \theta_{2} & 0 & 0 \\
0 & 0 & 0 & 1
\end{array}\right], \\
{ }_{3}^{2} T=\left[\begin{array}{cccc}
c \theta_{3} & -s \theta_{3} & 0 & \alpha_{3} \\
s \theta_{3} & c \theta_{3} & 0 & 0 \\
0 & 0 & 1 & 0 \\
0 & 0 & 0 & 1
\end{array}\right], \\
{ }_{4}^{3} T=\left[\begin{array}{cccc}
1 & 0 & 0 & \alpha_{4} \\
0 & 1 & 0 & 0 \\
0 & 0 & 1 & 0 \\
0 & 0 & 0 & 1
\end{array}\right],
\end{gathered}
$$

where, the angles $\theta_{1}, \theta_{2}$, and $\theta_{3}$ are depicted in Figure 6 on the left three-joint finger.

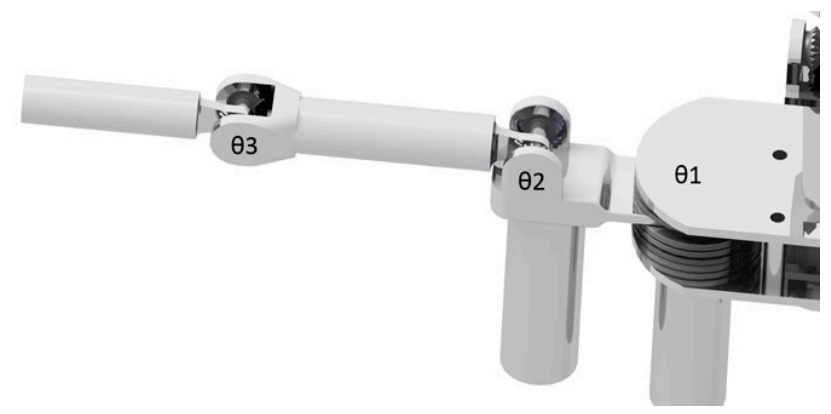

Figure 6. Angles $\theta_{1}, \theta_{2}$, and $\theta_{3}$ in the left three-joint finger.

Since the transformation matrices from one link to the next have been defined, it is now that the final transformation matrix (from the base to the fingertip), can be calculated by multiplying the matrices from the base link to the fingertip link, as shown below.

$$
{ }_{1}^{0} T_{2}^{1} T_{3}^{2} T_{4}^{3} T={ }_{4}^{0} T=\left[\begin{array}{cccc}
r_{11} & r_{12} & r_{13} & p_{x} \\
r_{21} & r_{22} & r_{23} & p_{y} \\
r_{31} & r_{32} & r_{33} & p_{z} \\
0 & 0 & 0 & 1
\end{array}\right]
$$

where:

$$
\begin{gathered}
r_{11}=c\left(\theta_{2}+\theta_{3}\right) c \theta_{1} \\
r_{12}=-s\left(\theta_{2}+\theta_{3}\right) c \theta_{1} \\
r_{13}=-s \theta_{1} \\
r_{21}=c\left(\theta_{2}+\theta_{3}\right) s \theta_{1}
\end{gathered}
$$




$$
\begin{gathered}
r_{22}=-s\left(\theta_{2}+\theta_{3}\right) s \theta_{1} \\
r_{23}=c \theta_{1} \\
r_{31}=-s\left(\theta_{2}+\theta_{3}\right) \\
r_{32}=-c\left(\theta_{2}+\theta_{3}\right) \\
r_{33}=0 \\
p_{x}=\alpha_{2} c \theta_{1}+\alpha_{3} c \theta_{1} c \theta_{2}+\alpha_{4} c \theta_{1} c\left(\theta_{2}+\theta_{3}\right)-d_{2} s \theta_{1} \\
p_{y}=a_{2} s \theta_{1}+\alpha_{3} s \theta_{1} c \theta_{2}+\alpha_{4} s \theta_{1} c\left(\theta_{2}+\theta_{3}\right)+d_{2} c \theta_{1} \\
p_{z}=-\alpha_{3} s \theta_{2}-\alpha_{4} s\left(\theta_{2}+\theta_{3}\right)
\end{gathered}
$$

Multiplying both sides of (16) by $\left(-s \theta_{1}\right)$ and both sides of $(17)$ by $\left(-c \theta_{1}\right)$, the system can be solved for $\theta_{1}$ :

$$
\theta_{1}=\operatorname{Atan} 2\left(4 p_{x} p_{y}+d_{2}^{2}+d_{2} \sqrt{d_{2}^{2}+4 p_{x} p_{y}+4 p_{x}^{2}}, 2 p_{x}^{2}\right)
$$

Similarly, multiplying (16) and (17) by $\left(c \theta_{1}\right)$ and $\left(s \theta_{1}\right)$, respectively, the value of $\theta_{2}$ is calculated as:

$$
\theta_{2}=\operatorname{Atan} 2\left(t^{2}+2 a_{2} a_{3}+t \sqrt{t^{2}+4 a_{2} a_{3}+4 a_{2}^{2}}, 2 a_{2}^{2}\right)
$$

where

$$
t=p_{x} c \theta_{1}+p_{y} s \theta_{1}-a_{2}
$$

We multiply again (16) and (17) by $\left(c \theta_{1}\right)$ and $\left(s \theta_{1}\right)$ solving for $c\left(\theta_{2}+\theta_{3}\right)$, and we solve (18) for $s\left(\theta_{2}+\theta_{3}\right)$. By dividing the two results, we obtain $\theta_{3}$ :

$$
\theta_{3}=\operatorname{Atan} 2\left(a_{3} s \theta_{2}-p_{z}, a_{2}+a_{3} c \theta_{2}-p_{x} c \theta_{1}-p_{y} s \theta_{1}\right)-\theta_{2}
$$

This way, the Inverse Kinematics of one of the three DoF fingers has been defined. Investigation of similar mechanism structure and inverse kinematics has been discussed in [37]. Figure 7 shows the kinematic model of the left three-DoF finger on a simple GUI for the Denavit-Hartenberg parameters robot arm design, as provided by the MRPT (Mobile Robot Programming Toolkit) [38].

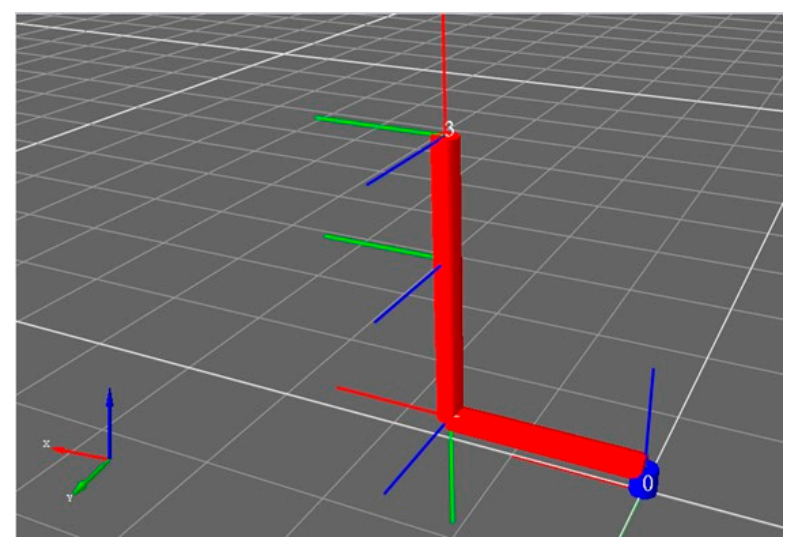

Figure 7. Kinematic model of the left three-DoF finger on a simple GUI for Denavit-Hartenberg parameters robot arm design.

The inverse kinematics has also been calculated and the kinematics of the gripper was visualized through Matlab with the assistance of the Robotics Toolbox [39]. Since each finger should be able to achieve a different position from the other ones for the grasping of an object, they are treated as a 
separate serial link, with a specified distance from the gripper base. As such, its inverse kinematics problem can be solved individually.

For testing and demonstration purposes, a random configuration has been considered. The gripper initial configuration, set in Matlab, is depicted in Figure 8a. After the final configuration has been calculated, the required trajectory was executed and ended up with the configuration requested by the user. The final configuration of the gripper can be seen in Figure $8 \mathrm{~b}$.

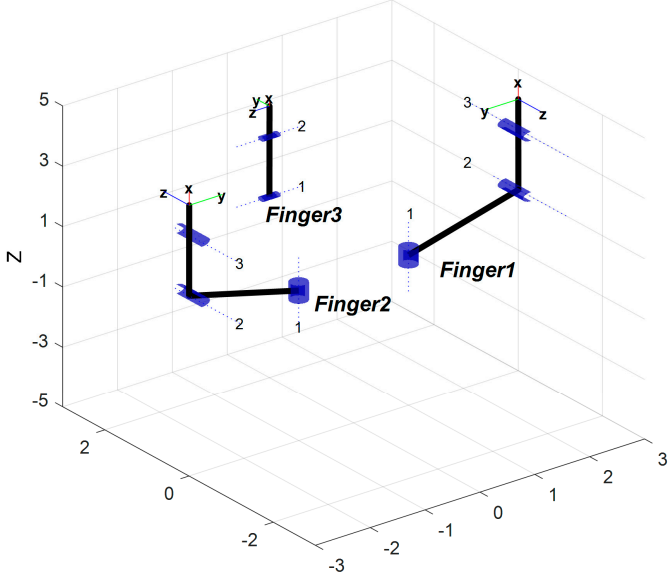

(a)

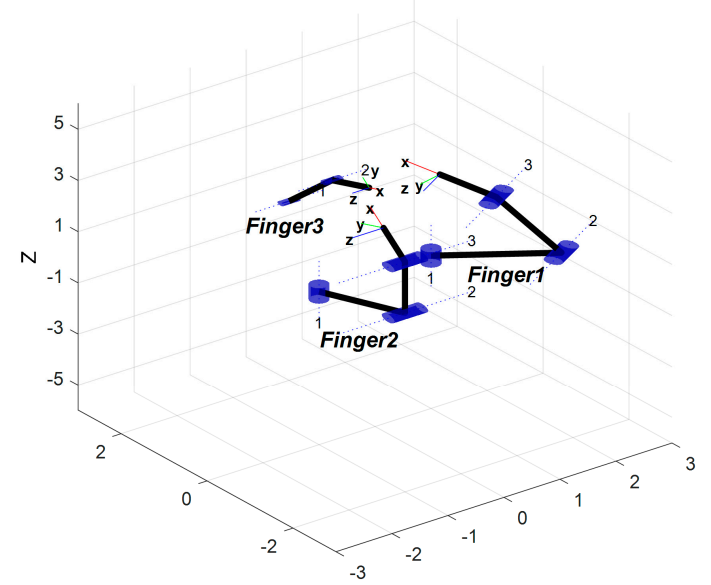

(b)

Figure 8. Matlab inverse kinematics simulation (a) before grasping and (b) after the position has been achieved.

In order to investigate the singularity points of the system we investigate the Jacobian (Equation (23) below):

$$
J=\left[\begin{array}{ccc}
-a_{2} s \theta_{1}-a_{3} s \theta_{1} c \theta_{2}-a_{4} s \theta_{1} c\left(\theta_{2}+\theta_{3}\right)-d_{2} c \theta_{1} & -a_{3} s \theta_{2} c \theta_{1}-a_{4} s\left(\theta_{2}+\theta_{3}\right) c \theta_{1} & -a_{4} s\left(\theta_{2}+\theta_{3}\right) c \theta_{1} \\
a_{2} c \theta_{1}+a_{3} c \theta_{1} c \theta_{2}+a_{4} c \theta_{1} c\left(\theta_{2}+\theta_{3}\right)-d_{2} s \theta_{1} & -a_{3} s \theta_{1} s \theta_{2}-a_{4} s\left(\theta_{2}+\theta_{3}\right) s \theta_{1} & -a_{4} s\left(\theta_{2}+\theta_{3}\right) s \theta_{1} \\
0 & -a_{3} c \theta_{2}-a_{4} c\left(\theta_{2}+\theta_{3}\right) & -a_{4} c\left(\theta_{2}+\theta_{3}\right)
\end{array}\right]
$$

Singularities occur where the determinant of the Jacobian equals zero

$$
\operatorname{det}=a_{3} a_{4}\left(a_{2}+a_{3} c \theta_{2}+a_{4} c\left(\theta_{2}+\theta_{3}\right)\right) s \theta_{3}
$$

Thus, the below conditions can be identified:

$$
\begin{gathered}
a_{3}=0 \text {, meaning zero link length for link } 3 \\
a_{4}=0 \text {, meaning zero link length for link } 4 \\
\theta_{3}=\left\{\begin{array}{c}
0 \\
\pi \\
\pm \frac{a \cos \left(-\left(a_{2}+a_{3} c \theta_{2}\right)\right)}{a_{4}}-\theta_{2}
\end{array}\right.
\end{gathered}
$$

\section{Results and Discussion}

This chapter is dedicated to presenting the gripper that was developed and the components that were used for its implementation. In Figure 9, the implemented gripper is shown in various grasping positions using two or three fingers in asymmetric positions to grasp parts that are often encountered in the automotive assembly and its suppliers. 


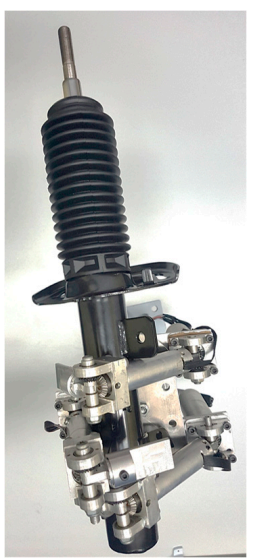

(a)

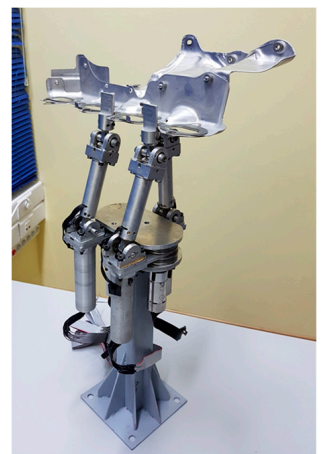

(e)

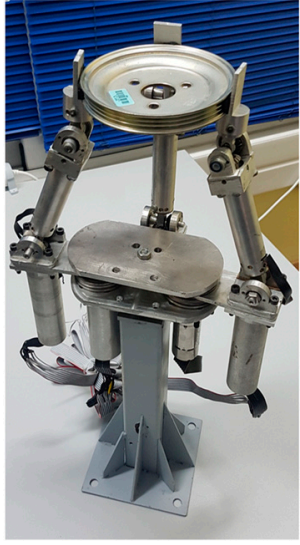

(b)

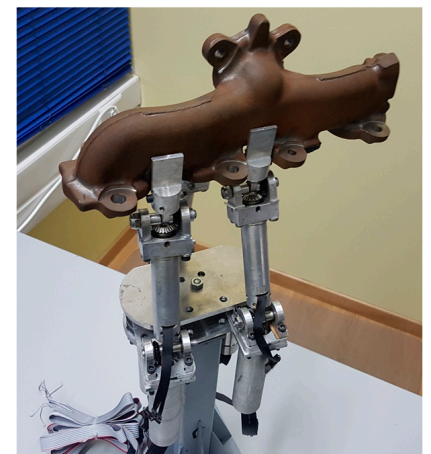

(f)

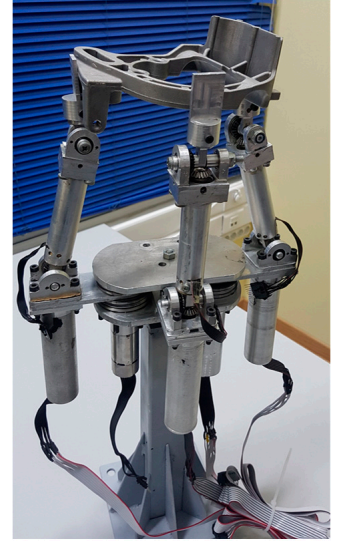

(c)

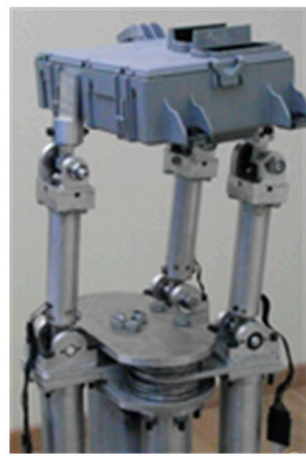

(g)

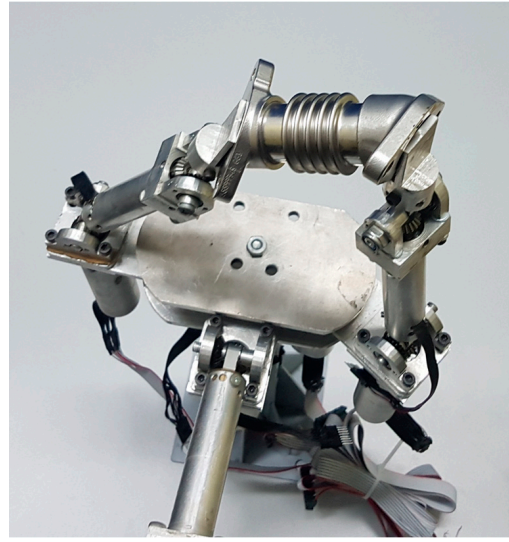

(d)

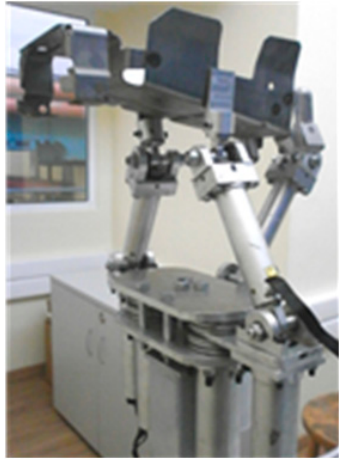

(h)

Figure 9. Gripper grasping positions grasping (a) suspension damper, (b) car pump pulley, (c) car pump carriage, (d) gas return, (e) heat shield, (f) manifold, (g) vehicle computer, and (h) car floor part.

The ability of the fingers to be individually configured thanks to the separately actuated joints, allows for the achievement of non-symmetric configurations both in terms of the finger joints, as well as the palm element, which eventually define the abduction/adduction motion. With this degree of flexibility, multiple configurations can be used to achieve similar grasping, where it would not have been possible with kinematically coupled joints.

\subsection{Actuation and Drive Electronics}

In order for the requirements of precise position and speed control as well as maximum output torque to be satisfied, brushless direct current (DC) servomotors have been selected thanks to their speed and efficiency in the most compact size. Table 3 summarizes the specifications of these actuators. These actuators are fitted with planetary gearheads and have integrated high resolution absolute encoders. Regarding size restrictions, the motors that control the fingertips are smaller, and therefore, less powerful than the rest of the actuators. Based on this selection of actuators, the specifications of the proposed gripper are presented in Table 4.

Table 3. Specifications of the actuators.

\begin{tabular}{ccc}
\hline Characteristic & Fingertip DoF & Middle and Base Finger DoF \\
\hline Motor type & DC brushless & DC brushless \\
Reduction rate & 25 & 29 \\
Max. speed (rpm) & 300 & 300 \\
Max. torque $(\mathrm{Nm})$ & 1.5 & 8 \\
Size $(\mathrm{mm})$ & $\Phi 22 \times 85$ & $\Phi 32 \times 100$ \\
Weight $(\mathrm{g})$ & 100 & 200 \\
Sensor resolution & 4096 & 4096 \\
\hline
\end{tabular}


Table 4. Specifications for the developed robotic hand.

\begin{tabular}{cc}
\hline Specification & Value \\
\hline Max. speed (at tip) $(\mathrm{m} / \mathrm{s})$ & $24\left({ }^{* 1}\right)$ \\
Max. force (at tip) $(\mathrm{N})$ & $55\left({ }^{* 2}\right)$ \\
Total DoF & 8 \\
Weight $(\mathrm{kg})$ & $\approx 3.5$ \\
Joint resolution $(\mathrm{deg})$ & 0.0035 or $0.003\left({ }^{* 3}\right)$ \\
\hline
\end{tabular}

${ }^{* 1}$ fingertip at 0 degrees and both finger motors output maximum speed; ${ }^{* 2}$ fingertip at 90 degrees and middle finger joint outputs maximum torque; $* 30.0035$ resolution for the smaller motors and 0.003 for the larger ones.

\subsection{Gripper Control}

The selected motors for the gripper have been provided by Faulhaber (www.faulhaber.com/) and are directly controlled by motion controllers that allow various operation modes: the speed, position, speed and acceleration curve, and voltage or current control. The motion controllers, apart from the aforementioned operation modes, are also used as a breaking mechanism, and therefore, there is no need for the motors to have mechanical breaks integrated. The motion controller communication protocol chosen is the CAN (Controller Area Network) bus, which has a lot of similarities with the older RS232, but allows for higher transfer rates of up to $1 \mathrm{Mbits} / \mathrm{s}$ (dependent on the wire length). The physical connection of the CAN is based on the logic that all of the nodes are connected in parallel and they are terminated at both ends with one $120 \mathrm{Ohm}$ resistor on each side. In this case, every motor is connected to its own motion controller and the controllers are connected to an external computer (see Figure 10).

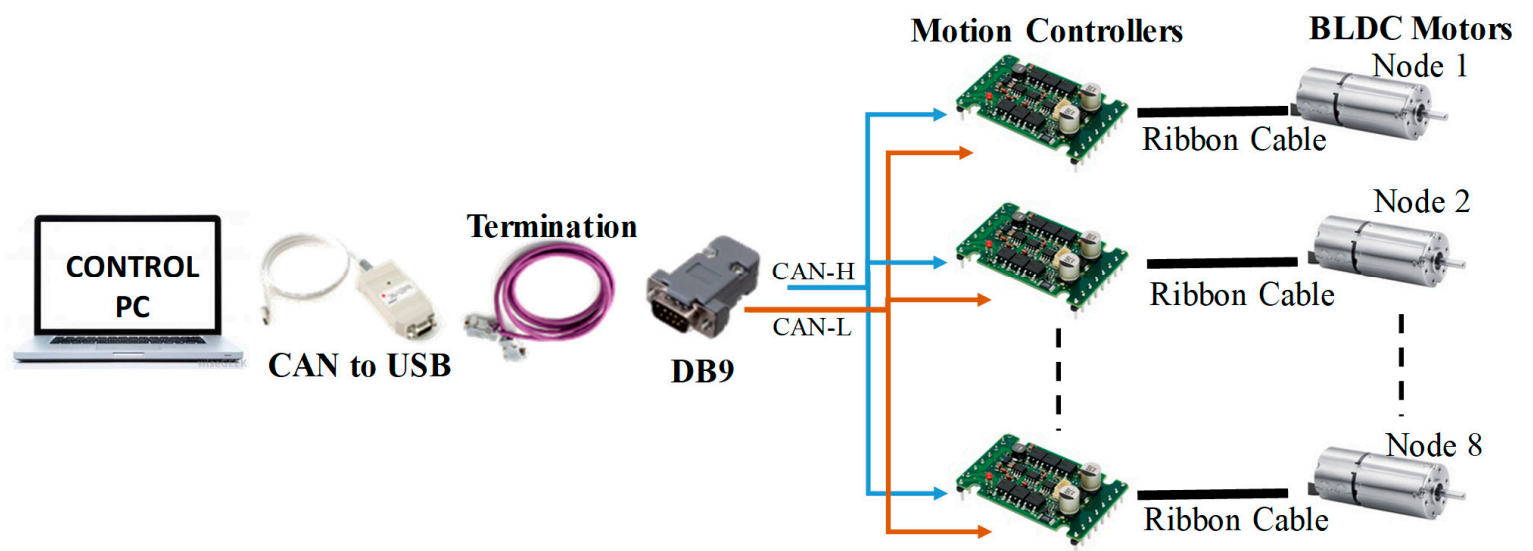

Figure 10. Control architecture diagram.

In order for any user to easily control the position of the gripper fingers, a custom Graphical User Interface has been developed. The GUI is based on the way the messages are transmitted on the PEAK's PCAN-Light API and it has been developed using visual C\#. Apart from the connection and message I/O parts of the software, as shown below in Figure 11, the main section responsible for the position control of the gripper's DOFs is the "Forward Control" section. The user can control the joints either by using the textboxes and pressing the execute position with an accuracy of two decimals, or use the sliders to move the joints at the same time with the positioning accuracy of one degree. The user is also allowed to record a group of positions in order to export them and use them, at a later instance, through importing the same file and selecting the position to be executed from the dropdown list. 


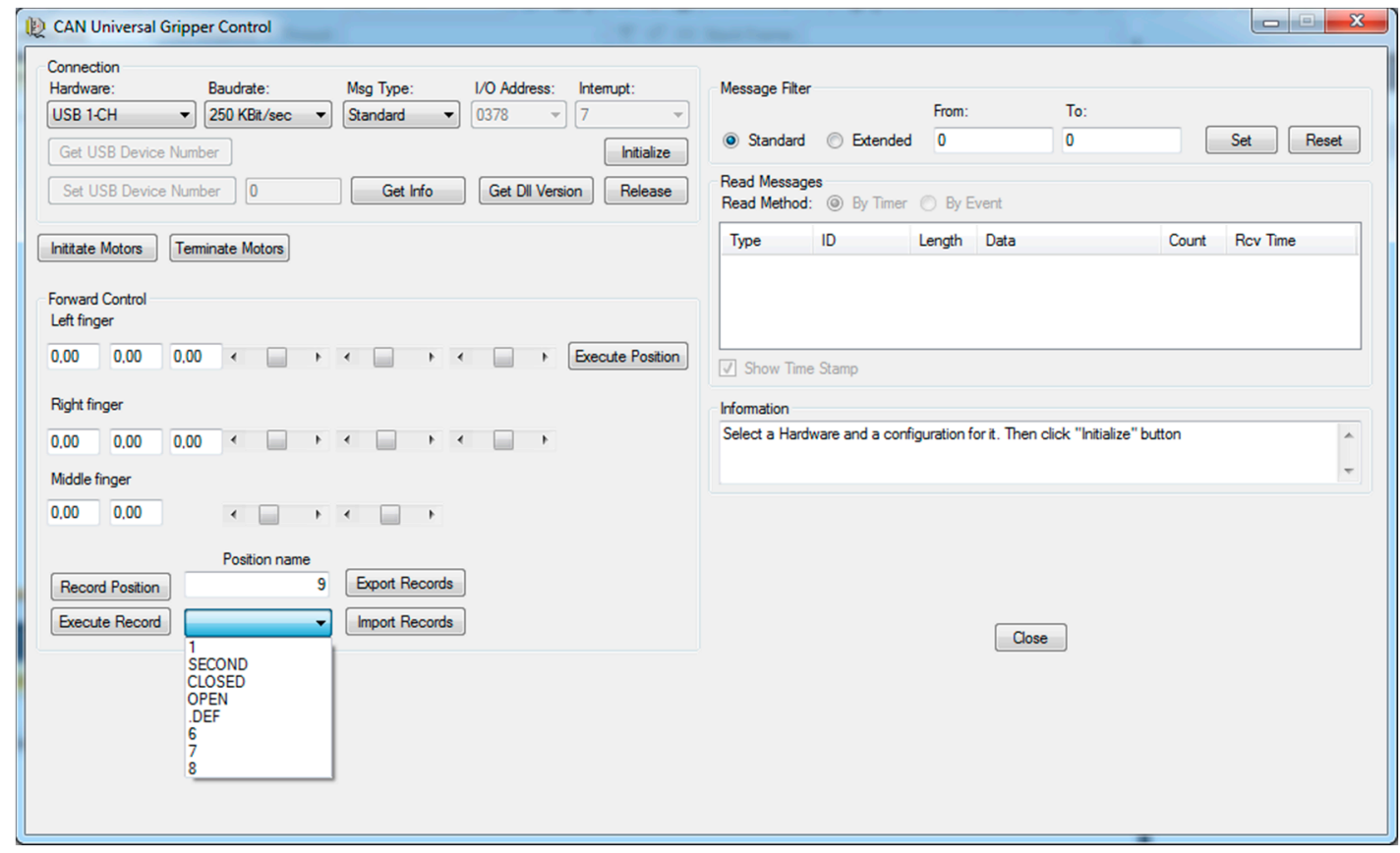

Figure 11. Control Graphical User Interface generic screenshot.

Having presented the design and implementation of the reconfigurable gripper, Table 5 provides a short comparison of the gripper against a set of the five most relevant three finger grippers identified in the literature.

Table 5. Comparison with other gripper specifications.

\begin{tabular}{ccccc}
\hline Gripper & $\begin{array}{c}\text { DoF } \\
\text { (Total/Actuated) }\end{array}$ & $\begin{array}{c}\text { Weight } \\
(\mathbf{k g})\end{array}$ & $\begin{array}{c}\text { Object Diameter for } \\
\text { Encompassing (mm) }\end{array}$ & $\begin{array}{c}\text { Maximum Payload } \\
\text { (Encompassing Grip) (N) }\end{array}$ \\
\hline Robotiq 3 Finger Gripper [40,41] & $10 / 4$ & 2.3 & $20-155$ & 100 \\
Schunk SDH [42] & $8 / 7$ & 1.95 & $17-215$ & N/A \\
Barret Hand [43,44] & $8 / 4$ & 1.2 & Up to 240 & 60 \\
LARM Hand IV [45] & $9 / 3$ & 1.5 & $10-100$ & N/A \\
Proposed Gripper & $8 / 8$ & 3.5 & Up to 215 & $>150$ \\
\hline
\end{tabular}

\section{Conclusions}

In this paper, a modular reconfigurable anthropomorphic gripper with multiple fingers that can execute high speed movements and grasp components weighing a few kilos, while keeping its design simple and its dimensions relatively compact, has been developed. The gripper has been tested for its capability of implementing on-demand reconfiguration of its fingers through the developed control modules. Different grasp types (pinching, enclosing, etc.) have been successfully tested, and more are considered possible with the use of customized finger tips.

Following the design and the construction of the first prototype, future work will focus on the experimental evaluation to ensure that it can sufficiently achieve the force/torque and speed values that are considered in the design stage. Furthermore, the implementation of a system that would provide feedback to the gripper's executed motion, and correct any mistakes, should be the next goal. Finally, automatic grasp planning algorithms should be developed to facilitate the automated and optimized grasping of different objects.

Acknowledgments: This study was partially supported by the projects: AUTORECON/FoF-ICT-285189 (http://www.autorecon.eu/) and VERSATILE/H2020-ICT-2016-731330 (http://www.versatile-project.eu) funded by the European Commission. 
Author Contributions: George Michalos, Jason Spiliotopoulos and Sotiris Makris conceived and designed the gripper device; Sotiris Makris provided the design of experiments and validation procedures; Jason Spiliotopoulos performed the construction and programming of the gripper; Sotiris Makris and George Michalos validated the results and performed the comparative analysis with the state of the art; Jason Spiliotopoulos, George Michalos and Sotiris Makris contributed to the writing of the paper.

Conflicts of Interest: The authors declare not conflicts of interest.

\section{References}

1. Chryssolouris, G. Manufacturing Systems: Theory and Practice, 2nd ed.; Mechanical Engineering Series; Springer: New York, NY, USA, 2006; ISBN 978-0-387-25683-2.

2. Krüger, J.; Wang, L.; Verl, A.; Bauernhansl, T.; Carpanzano, E.; Makris, S.; Fleischer, J.; Reinhart, G.; Franke, J.; Pellegrinelli, S. Innovative control of assembly systems and lines. CIRP Ann. Manuf. Technol. 2017, 66, 707-730. [CrossRef]

3. Michalos, G.; Makris, S.; Papakostas, N.; Mourtzis, D.; Chryssolouris, G. Automotive assembly technologies review: Challenges and outlook for a flexible and adaptive approach. CIRP J. Manuf. Sci. Technol. 2010, 2, 81-91. [CrossRef]

4. Pellegrinelli, S.; Pedrocchi, N.; Tosatti, L.M.; Fischer, A.; Tolio, T. Multi-robot spot-welding cells for car-body assembly: Design and motion planning. Robot. Comput. Integr. Manuf. 2017, 44, 97-116. [CrossRef]

5. Michalos, G.; Makris, S.; Chryssolouris, G. The new assembly system paradigm. Int. J. Comput. Integr. Manuf. 2015, 28, 1252-1261. [CrossRef]

6. Makris, S.; Michalos, G.; Eytan, A.; Chryssolouris, G. Cooperating robots for reconfigurable assembly operations: Review and challenges. Procedia CIRP 2012, 3, 346-351. [CrossRef]

7. Makris, S.; Michalos, G.; Chryssolouris, G. Virtual commissioning of an assembly cell with cooperating robots. Adv. Decis. Sci. 2012, 2012,1-11. [CrossRef]

8. Papakostas, N.; Michalos, G.; Makris, S.; Zouzias, D.; Chryssolouris, G. Industrial applications with cooperating robots for the flexible assembly. Int. J. Comput. Integr. Manuf. 2011, 24, 650-660. [CrossRef]

9. Tsarouchi, P.; Matthaiakis, S.-A.; Michalos, G.; Makris, S.; Chryssolouris, G. A method for detection of randomly placed objects for robotic handling. CIRP J. Manuf. Sci. Technol. 2016, 14, 20-27. [CrossRef]

10. Fantoni, G.; Santochi, M.; Dini, G.; Tracht, K.; Scholz-Reiter, B.; Fleischer, J.; Kristoffer Lien, T.; Seliger, G.; Reinhart, G.; Franke, J.; et al. Grasping devices and methods in automated production processes. CIRP Ann. Manuf. Technol. 2014, 63, 679-701. [CrossRef]

11. Schunk Parallel Gripper. Available online: https://schunk.com/de_en/gripping-systems/category/gripp ing-systems/schunk-grippers/parallel-gripper/ (accessed on 5 November 2017).

12. Schunk Centric Gripper. Available online: https://schunk.com/br_en/gripping-systems/category/grippin g-systems/schunk-grippers/centric-gripper/ (accessed on 5 November 2017).

13. Adaptive Robot Gripper 2-Finger. Available online: http://robotiq.com/products/adaptive-robot-gripper/ (accessed on 5 November 2017).

14. Townsend, W. The BarrettHand grasper-Programmably flexible part handling and assembly. Ind. Robot Int. J. 2000, 27, 181-188. [CrossRef]

15. Carbone, G.; González, A. A numerical simulation of the grasp operation by LARM Hand IV: A three finger robotic hand. Robot. Comput. Integr. Manuf. 2011, 27, 450-459. [CrossRef]

16. Fukaya, N.; Toyama, S.; Asfour, T.; Dillmann, R. Design of the TUAT/Karlsruhe humanoid hand. In Proceedings of the IEEE/RSJ International Conference on Intelligent Robots and Systems (IROS 2000), Takamatsu, Japan, 30 October-5 November 2000; Volume 3, pp. 1754-1759.

17. Kappassov, Z.; Khassanov, Y.; Saudabayev, A.; Shintemirov, A.; Varol, H.A. Semi-anthropomorphic 3D printed multigrasp hand for industrial and service robots. In Proceedings of the IEEE International Conference on Mechatronics and Automation (ICMA), Takamatsu, Japan, 4-7 August 2013; pp. 1697-1702.

18. Robotic Hands (Self-contained) Prensilia s.r.l. Available online: http://www.prensilia.com/index.php?q=en / node/40 (accessed on 21 July 2017). 
19. Salisbury, J.K.; Craig, J.J. Articulated hands: Force control and kinematic issues. Int. J. Robot. Res. 1982, 1, 4-17. [CrossRef]

20. Jacobsen, S.; Iversen, E.; Knutti, D.; Johnson, R.; Biggers, K. Design of the Utah/M.I.T. Dextrous Hand. In Proceedings of the IEEE International Conference on Robotics and Automation, San Francisco, CA, USA, 7-10 April 1986; Volume 3, pp. 1520-1532.

21. Lovchik, C.S.; Diftler, M.A. The Robonaut hand: A dexterous robot hand for space. In Proceedings of the IEEE International Conference on Robotics and Automation, Detroit, MI, USA, 10-15 May 1999; Volume 2, pp. 907-912.

22. Zhang, Y.; Han, Z.; Zhang, H.; Shang, X.; Wang, T.; Guo, W.; Gruver, W.A. Design and control of the BUAA four-fingered hand. In Proceedings of the ICRA IEEE International Conference on Robotics and Automation, Seoul, Korea, 21-26 May 2001; Volume 3, pp. 2517-2522.

23. Lin, L.-R.; Huang, H.-P. NTU hand: A new design of dexterous hands. J. Mech. Des. 1998, 120, 282. [CrossRef]

24. Kawasaki, H.; Komatsu, T.; Uchiyama, K.; Kurimoto, T. Dexterous anthropomorphic robot hand with distributed tactile sensor: Gifu hand II. In Proceedings of the IEEE International Conference on Systems, Man, and Cybernetics, Tokyo, Japan, 12-15 October 1999; Volume 2, pp. 782-787.

25. Mouri, T.; Kawasaki, H.; Ito, S. Unknown object grasping strategy imitating human grasping reflex for anthropomorphic robot hand. J. Adv. Mech. Des. Syst. Manuf. 2007, 1, 1-11. [CrossRef]

26. Butterfass, J.; Grebenstein, M.; Liu, H.; Hirzinger, G. DLR-Hand II: Next generation of a dextrous robot hand. In Proceedings of the ICRA IEEE International Conference on Robotics and Automation, Seoul, Korea, 21-26 May 2001; Volume 1, pp. 109-114.

27. Melchiorri, C.; Palli, G.; Berselli, G.; Vassura, G. Development of the UB hand IV: Overview of design solutions and enabling technologies. IEEE Robot. Autom. Mag. 2013, 20, 72-81. [CrossRef]

28. Dexterous Hand-Shadow Robot Company. Available online: http://www.shadowrobot.com/products/d exterous-hand/ (accessed on 5 November 2017).

29. SDH. Available online: https://schunk.com/ch_en/gripping-systems/series/sdh/ (accessed on 5 November 2017).

30. elumotion.com: EH2-Elumotion Hand 2. Available online: http://www.elumotion.com/index.php/portfo lio/project-title-1 (accessed on 21 July 2017).

31. Namiki, A.; Imai, Y.; Ishikawa, M.; Kaneko, M. Development of a high-speed multifingered hand system and its application to catching. In Proceedings of the IEEE/RSJ International Conference on Intelligent Robots and Systems, Las Vegas, NV, USA, 27-31 October 2003; Volume 3, pp. 2666-2671.

32. Luo, M.; Carbone, G.; Ceccarelli, M.; Zhao, X. Analysis and design for changing finger posture in a robotic hand. Mech. Mach. Theory 2010, 45, 828-843. [CrossRef]

33. Cutkosky, M.R. On grasp choice, grasp models, and the design of hands for manufacturing tasks. IEEE Trans. Robot. Autom. 1989, 5, 269-279. [CrossRef]

34. Mohammadali, S.; Kambiz, G.O.; Amir Ali, A.K. Modular framework kinematic and fuzzy reward reinforcement learning analysis of a radially symmetric six-legged robot. Life Sci. J. 2013, 10, 120-129.

35. Saha, S.K. Introduction to Robotics; Tata McGraw-Hill: New Delhi, India, 2008; ISBN 978-0-07-066900-0.

36. Kucuk, S.; Bingul, Z. Robot Kinematics: Forward and Inverse Kinematics. In Industrial Robotics: Theory, Modelling and Control; Cubero, S., Ed.; Pro Literatur Verlag: Augsburg, Germany; ARS: Linz, Austria, 2006; ISBN 978-3-86611-285-8.

37. Lu, Z.; Xu, C.; Pan, Q.; Zhao, X.; Li, X. Inverse kinematic analysis and evaluation of a robot for nondestructive testing application. J. Robot. 2015, 2015, 596327. [CrossRef]

38. MRPT-Empowering C++ Development in Robotics. Available online: http://www.mrpt.org/ (accessed on 5 November 2017).

39. Corke, P.I. A robotics toolbox for MATLAB. IEEE Robot. Autom. Mag. 1996, 3, 24-32. [CrossRef]

40. Robotiq 3 Finger Adaptive Robot Gripper. Available online: https:/ / robotiq.com/products/3-finger-adapti ve-robot-gripper (accessed on 5 November 2017).

41. Franchi, G.; Hauser, K. Technical Report: Use of Hybrid Systems to model the RobotiQ Adaptive Gripper. Available online: https:/ / www.cs.indiana.edu/ftp/techreports/TR711.pdf (accessed on 5 November 2017).

42. SCHUNK Dextrous Hand 2.0 (SDH 2.0). Available online: https:/ /www.nist.gov/sites/default/files/doc uments /2017/05/09/9020173R1.pdf (accessed on 5 November 2017). 
43. Lauzier, N. Barrett Hand vs. Robotiq Adaptive Gripper. 2012. Available online: https://blog.robotiq.com/b id/52340 / Barrett-Hand-vs-Robotiq-Adaptive-Gripper (accessed on 5 November 2017).

44. Barret Hand. Available online: http://www.barrett.com/products-hand-specifications.htm (accessed on 5 November 2017).

45. Larm Hand/ARMAND. Available online: http://www.mechanimata.it/flyers/ARMAND.pdf (accessed on 5 November 2017). 